\title{
High expression of CXCR4 and stem cell markers in a monocrotaline and chronic hypoxia-induced rat model of pulmonary arterial hypertension
}

\author{
TINGTING ZHANG, NANAKO KAWAGUCHI, EMIKO HAYAMA, \\ YOSHIYUKI FURUTANI and TOSHIO NAKANISHI \\ Department of Pediatric Cardiology, Tokyo Women's Medical University, Tokyo 162-8666, Japan
}

Received October 4, 2017; Accepted February 9, 2018

DOI: $10.3892 /$ etm.2018.6027

\begin{abstract}
Pulmonary arterial hypertension (PAH) is a severe and fatal clinical syndrome. C-X-C chemokine receptor type 4 (CXCR4) is known to serve a key role in recruiting mesenchymal stem cells (MSCs) from the bone marrow. In the present study, a rat model of PAH induced by 5 weeks of chronic hypoxia and treatment with a single injection of monocrotaline $(60 \mathrm{mg} / \mathrm{kg})$ was used to investigate the involvement of CXCR4 in PAH. Successful establishment of the PAH model was confirmed by significant differences between the $\mathrm{PAH}$ and control groups in right ventricular systolic pressure, Fulton index, wall thickness, vascular occlusion score determined by immunohistochemical staining and the expression of inflammatory markers measured by reverse transcription-quantitative polymerase chain reaction (RT-qPCR). The expression of CXCR4 and other stem cell markers were compared in the PAH and control groups. RT-qPCR showed that the expression of CXCR4, SCF, c-Kit, and CD29, which are expressed in MSCs, was significantly higher in the PAH group. Immunohistochemical staining also showed that the numbers of CXCR4-, c-Kit- and CD90-positive cells were significantly higher in the PAH group. These results suggest that CXCR4 is involved in the pathogenesis of PAH and that stem cells may serve an important role in pulmonary vascular remodeling.
\end{abstract}

\section{Introduction}

Pulmonary arterial hypertension (PAH) is a severe and fatal clinical syndrome that can cause high blood pressure in the

Correspondence to: Professor Toshio Nakanishi, Department of Pediatric Cardiology, Tokyo Women's Medical University, 8-1 Kawada-cho, Shinjuku, Tokyo 162-8666, Japan

E-mail: pnakanis@hij.twmu.ac.jp

Key words: pulmonary arterial hypertension, CXCR4, mesenchymal stem cells, pulmonary vascular remodeling, proliferation, pulmonary arteriolar occlusion pulmonary artery via right ventricular overload, right heart failure, and death $(1,2)$. The pathogenesis of PAH is complex and known to involve endothelial dysfunction, proliferation of smooth muscle cells, pulmonary arteriolar occlusion, chronic inflammation, and pulmonary vascular remodeling (3-6). Three main classes of drugs have been widely used to treat PAH: Endothelin-1 receptor antagonists, phosphodiesterase type 5 inhibitors, and prostacyclins. However, these drugs can only delay the course of PAH and cannot cure the disease (7). Recent studies of PAH treatment have focused on gene and cell therapy in animal models and in some clinical cases, as these treatments are considered safe and can attenuate pulmonary vascular remodeling and right ventricular hypertrophy (8-10). Gene and cell therapy are intended to promote angiogenesis, increase blood flow, and alleviate ischemia.

C-X-C chemokine receptor type 4 (CXCR4) is known to be expressed in cancer cells and stem/progenitor cells, including endothelial and smooth muscle progenitors $(11,12)$. The ligand of CXCR4, stromal cell derived factor-1 (SDF-1), recruits CXCR4-expressing cells to SDF-1-expressing cells at sites of ischemic injury.

CXCR4 inhibitors have been used to investigate the function of CXCR4 and inhibit tumor cell migration and proliferation (13). Furthermore, inhibition of CXCR4 using small-molecule inhibitors can prevent pulmonary arterial muscularization in PAH models (14). Mesenchymal stem cells (MSCs) and gene therapies have emerged as novel tools for the treatment of PAH (2).

The purpose of this study was to investigate the involvement of CXCR4 and stem cells such as MSCs in a monocrotaline (MCT) and chronic hypoxia $(\mathrm{CH})$-induced model of PAH by measuring the gene/protein expression levels of CXCR4 and stem cell/MSC marker genes and proteins. We focused on MSCs from bone marrow, which can differentiate into endothelial and smooth muscle cells. Upregulation of CXCR4 and MSC markers in PAH models would suggest that CXCR4 is involved in the development of PAH. Excessive CXCR4 recruitment of MSCs may cause MSCs not to fully differentiate into endothelial and smooth muscle cells, or other lung cells, which may impair lung tissues and vessels and lead to PAH. In this case, CXCR4 inhibitors may be useful 
for treating $\mathrm{PAH}$, as inhibition of CXCR4 would inhibit the recruitment of MSCs.

\section{Materials and methods}

Animal models. Male 6-week-old Sprague-Dawley rats (180-230 g; Tokyo Experimental Animal Company, Tokyo, Japan) were randomly assigned into two groups with eight rats/group. PAH model rats were established as previously described (10). Briefly, i) control group, rats were subcutaneously injected with a single dose of $0.9 \%$ saline and maintained in a chamber with normal air for 5 weeks; ii) PAH group, rats were subcutaneously injected with a single dose of MCT and maintained in a hypoxic chamber for 5 weeks. The oxygen concentration was maintained at $10 \%$ by continuously flushing the chamber with a gas mixture of low $\% \mathrm{O}_{2}$ and high $\% \mathrm{~N}_{2}$. MCT (Sigma-Aldrich; Merck KGaA, Darmstadt, Germany) was dissolved in $1 \mathrm{~N} \mathrm{HCl}$, neutralized with $1 \mathrm{~N} \mathrm{NaOH}$, and diluted with distilled water to $20 \mathrm{mg} / \mathrm{ml}$. A dose of $60 \mathrm{mg} / \mathrm{kg}$ ( $3 \mathrm{ml} / \mathrm{kg}$ ) was administered to the animals $(10,15)$. All rats had unlimited access to food and water and were weighed weekly.

All experiments were conducted according to a protocol approved by the Institutional Animal Experiment Committee of the Tokyo Women's Medical University (AE16-117). All applicable international, national, and/or institutional guidelines for the care and use of animals were followed. All procedures performed in studies involving animals were in accordance with the ethical standards of the institution.

Hemodynamic studies and evaluation of right ventricular hypertrophy. Five weeks after MCT injection, the rats were anesthetized by isoflurane inhalation. A micro-tip catheter (Millar Instruments, Houston, TX, USA) was inserted into the right ventricle $(\mathrm{RV})$ via the right jugular vein to measure the right ventricular systolic pressure (RVSP). The catheter was connected to a PowerLab Data Acquisition system and Lab Chart 7 software (ADInstruments, Dunedin, New Zealand), which were used to record the data. After hemodynamic evaluation, the rats were sacrificed by bloodletting. The heart, lungs, and pulmonary arteries (PAs) were separated and harvested, and then the free wall of the RV was separated and weighed. The left ventricle and septum $(\mathrm{LV}+\mathrm{S})$ were also separated and weighed. The Fulton index (weight ratio of $\mathrm{RV}$ and $\mathrm{LV}+\mathrm{S}$ ) was measured $(10,15)$.

Immunohistochemical staining. The left and right lower lobes of the rat lungs were fixed by tracheal infusion of $4 \%$ paraformaldehyde ( $\mathrm{pH} 7.4$ ) and incubated in $4 \%$ paraformaldehyde overnight. Lungs were prepared as paraffin-embedded tissue samples and cut into $4-\mu \mathrm{m}$-thick sections. After deparaffinizing, some sections were stained with hematoxylin and eosin (H\&E). Antigen retrieval was achieved by treating the sections with Immunosaver (Nisshin EM Co., Ltd., Tokyo, Japan) at $98^{\circ} \mathrm{C}$ for $45 \mathrm{~min}$ in a kitchen electric pot (Zojirushi Corporation, Osaka, Japan; CD-WU30). After incubation with $0.3 \% \mathrm{H}_{2} \mathrm{O}_{2}$ for 30 min to block endogenous peroxidase activity, the sections were treated with normal goat serum for $30 \mathrm{~min}$, and then incubated with primary antibodies at $4^{\circ} \mathrm{C}$ overnight. Immunohistochemical staining was conducted using antibodies against $\alpha$-smooth muscle actin ( $\alpha$-SMA)
(1:500; Sigma-Aldrich; Merck KGaA), proliferating cell nuclear antigen (PCNA; 1:125; Sigma-Aldrich; Merck KGaA), c-Kit (1:50; Santa Cruz Biotechnology, Inc., Dallas, TX, USA), CD90 (1:50; Santa Cruz Biotechnology, Inc.), and CXCR4 (1:500; Abcam, Cambridge, UK) as primary antibodies. The sections were further incubated with a biotinylated secondary antibody at room temperature for $1 \mathrm{~h}$, and then with avidin-biotin complex (Vector Laboratories, Peterborough, UK), followed by diaminobenzidine (Nacalai Tesque, Kyoto, Japan). Mayer's hematoxylin (Wako Pure Chemical Industries, Ltd., Osaka, Japan) was used for counterstaining.

$\alpha$-SMA staining was used to calculate the percent medial wall thickness (\%MT). The external diameter (ED) and MT were measured in muscularized PAs, whose EDs varied from 50 to $100 \mu \mathrm{m}$, to calculate $\% \mathrm{MT}=(2 \mathrm{x} \mathrm{MT} / \mathrm{ED}) \mathrm{x} 100$ (16). For all evaluations, 20 intra-acinar PAs per section from each rat were randomly selected. PCNA staining was used to calculate the vascular occlusion score (VOS), which was categorized as Grade 0 (no evidence of neointimal formation), Grade 1 (less than $50 \%$ luminal occlusion), or Grade 2 (more than $50 \%$ luminal occlusion) (17). For all evaluations, 20 intra-acinar PAs per section from each rat were randomly selected.

CXCR4 staining was performed to compare expression of this protein in PAH rats and control rats, and CD90 and c-Kit staining were performed to compare the number of stem cells in PAH rats and control rats. Because CXCR4-positive cells aggregated, it was impractical to count individual cells; thus, the number of CXCR4-positive cell aggregates was counted instead. The numbers of c-Kit and CD90-positive cells around PAs were counted and compared between the two groups. For each type of staining, we randomly chose 15-20 microscopic areas from each rat.

$R T-q P C R$. RNA was isolated from the small PA and surrounding lung tissue in the left and right lower lobes of the lung using the RNeasy Mini kit (Qiagen, Hilden, Germany). cDNA was synthesized using the PrimeScript ${ }^{\mathrm{TM}}$ RT Reagent kit (Takara Bio, Shiga, Japan). qPCR was performed using a Thermo Scientific PikoReal Real-Time PCR System (Thermo Fisher Scientific, Inc., Waltham, MA, USA). Each sample was analyzed in triplicate. $\beta$-actin mRNA expression was measured for normalization. mRNA expression was normalized to $\beta$-actin expression using the equation $2^{-\Delta \Delta \mathrm{Cq}}$ (18). Primer sequences are listed in Table I.

Statistical analysis. Quantitative data are expressed as the mean \pm standard deviation (SD). Statistical analyses were performed using the independent samples t-test in SPSS software (SPSS, Inc., Chicago, IL, USA). P $<0.05$ was considered to indicate a statistically significant difference.

\section{Results}

Animal survival rate, RVSP, and right ventricular hypertrophy measurement. The eight rats in the control group survived and remained active during the experiment. In contrast, two of the eight rats in the PAH group died during the experiment. The first died at 3 weeks and 5 days and the second died at 4 weeks and 4 days. Therefore, eight control rats and six PAH rats were used for the experiments described below. 
Table I. Primers used for reverse transcription-quantitative polymerase chain reaction.

\begin{tabular}{llll}
\hline Gene & \multicolumn{1}{c}{ Forward primer } & Reverse primer & Accession no. \\
\hline MCP1 & 5'-AGCATCCACGTGCTGTCTC-3' & 5'-GATCATCTTGCCAGTGAATGAG-3' & AY357296 \\
IL-6 & 5'-CCGGAGAGGAGACTTCACAG-3' & 5'-ACAGTGCATCATCGCTGTTC-3' & NG_011640 \\
TNF $\alpha$ & 5'-TGACCCCCATTACTCTGACC-3' & 5'-GGCCACTACTTCAGCGTCTC-3' & KY038170 \\
CXCR1 & 5'-GTCGTCATCTATGCCCTGGT-3' & 5'-GCCAGGTTCAGCAGGTAGAC-3' & NG_011814 \\
CXCR2 & 5'-CGCTCCGTCACTGATGTCTA-3' & 5'-GAGTGAGACCACCTTGCACA-3' & NG_052975 \\
CXCR4 & 5'-GCTGAGGAGCATGACAGACA-3' & 5'-GATGAAGGCCAGGATGAGAA-3' & NG_011587 \\
SCF & 5'-TCGTGGCATGTATGGAAGAA-3' & 5'-TCAGATGCCACCATGAAGTC-3' & KR815359 \\
c-Kit & 5'-GATCTGCTCTGCGTCCTGTT-3' & 5'-AGATGGCTGAGAAGTCCCTGT-3' & NG_007456 \\
CD29 & 5'-AACTGCACCAGCCCATTTAG-3' & 5'-CCACCTTCTGGAGAATCCAA-3' & NG_029012 \\
3-actin & 5'-CTAAGGCCAACCGTGAAAAG-3' & 5'-GCCTGGATGGCTACGTACA-3' & NM_031144
\end{tabular}

MCP1, monocyte chemoattractant protein 1; IL-6, interleukin-6; TNF $\alpha$, tumor necrosis factor $\alpha$; CXCR1, C-X-C chemokine receptor type 1; CXCR2, C-X-C chemokine receptor type 2 ; SCF, stem cell factor; CD, cluster of differentiation.
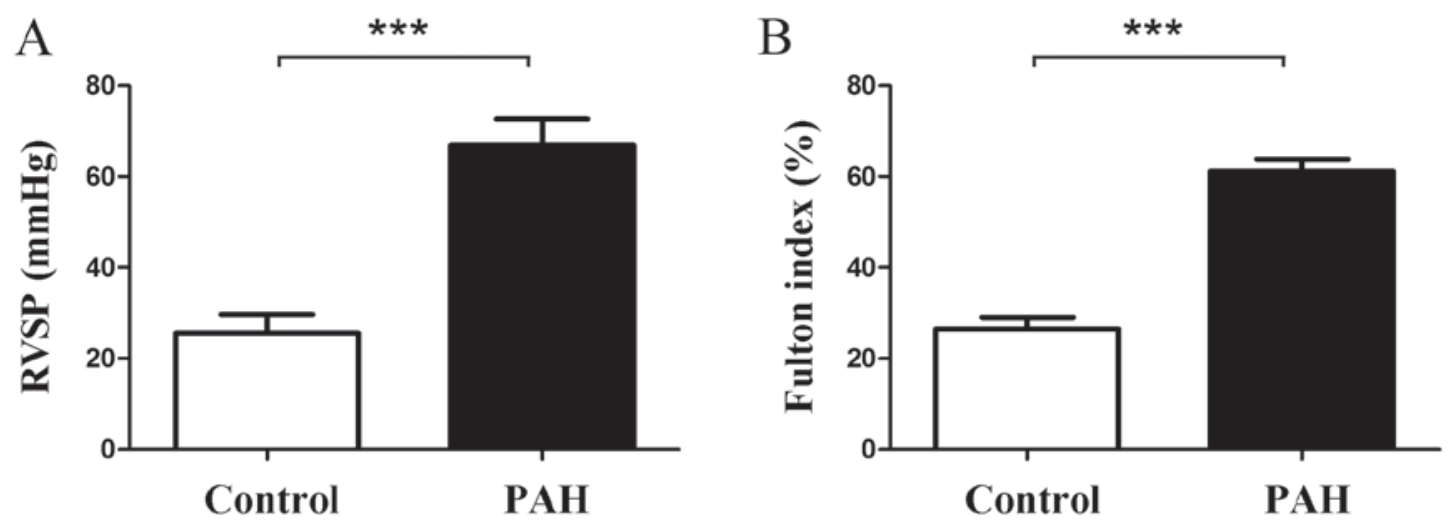

Figure 1. Hemodynamic studies of PAH models and normal rats. Comparison of (A) RVSP and (B) Fulton index between the two groups. ${ }^{* * * *} \mathrm{P}<0.001$. RVSP, right ventricular systolic pressure; PAH, pulmonary arterial hypertension.

Rats in the PAH group exhibited a significant increase in RVSP and Fulton index compared with the control group. RVSP was $66.91 \pm 5.76 \mathrm{mmHg}$ in the $\mathrm{PAH}$ group and $25.48 \pm 4.25 \mathrm{mmHg}$ in the control group ( $\mathrm{P}<0.001$, Fig. $1 \mathrm{~A})$; the Fulton index was $61.16 \pm 2.72 \%$ in the PAH group and $26.43 \pm 2.65 \%$ in the control group $(\mathrm{P}<0.001$, Fig. 1B).

Histopathology. \%MT and VOS. H\&E staining was performed to observe differences in the pulmonary artery wall between the control group (Fig. 2A) and the PAH group (Fig. 2B). To confirm that the thicker tissue consisted of smooth muscle cells, lung tissue sections were immunohistochemically stained using an $\alpha$-SMA antibody. Compared with the control group (Fig. 2C), increased proliferation of smooth muscle cells was observed in the PAH group (Fig. 2D). PCNA-positive proliferating cells were barely visible in the control group (Fig. 2E), but were distributed throughout the lumen and wall of the PA in the PAH group (Fig. 2F). Measurement of the thickness of the pulmonary artery wall indicated a significant difference in \%MT between the PAH group and the control group: $32.08 \pm 5.49 \%$ in the PAH group and $17.52 \pm 0.61 \%$ in the control group ( $\mathrm{P}=0.002$, Fig. $2 \mathrm{G}$ ). Furthermore, a significant difference was observed in VOS between the PAH and control groups: $1.66 \pm 0.28$ for the $\mathrm{PAH}$ group and 0 for the control group $(\mathrm{P}=0.001$, Fig. 2H).

Expression of inflammatory markers in RT-qPCR. mRNA expression was quantified relative to $\beta$-actin mRNA using the equation $2^{-\Delta \Delta C q}$. Rats in the PAH group exhibited significantly higher gene expression levels of MCP1 (relative expression $0.0439 \pm 0.0372$ in the PAH group and $0.0029 \pm 0.0007$ in the control group, $\mathrm{P}=0.017$, Fig. 3A), IL-6 $(0.0256 \pm 0.0225$ in the PAH group and $0.0032 \pm 0.0017$ in the control group, $\mathrm{P}=0.026$, Fig. 3B), TNFa $(0.1942 \pm 0.1940$ in the PAH group and $0.0172 \pm 0.0047$ in the control group, $\mathrm{P}=0.036$, Fig. 3C), CXCR1 $(0.00072 \pm 0.00058$ in the PAH group and $0.00013 \pm 0.00010$ in the control group, $P=0.037$, Fig. 3D), and CXCR2 $(0.0120 \pm 0.0080$ in the PAH group and $0.0011 \pm 0.0015$ in the control group, $\mathrm{P}=0.036$, Fig. 3E).

Expression of stem cell markers in $R T-q P C R$. Rats in the PAH group exhibited significantly higher gene expression levels of CXCR4 (relative expression $0.2438 \pm 0.2463$ in the PAH group and $0.0183 \pm 0.0131$ in the control group, $\mathrm{P}=0.036$, Fig. $4 \mathrm{~A}$ ), 


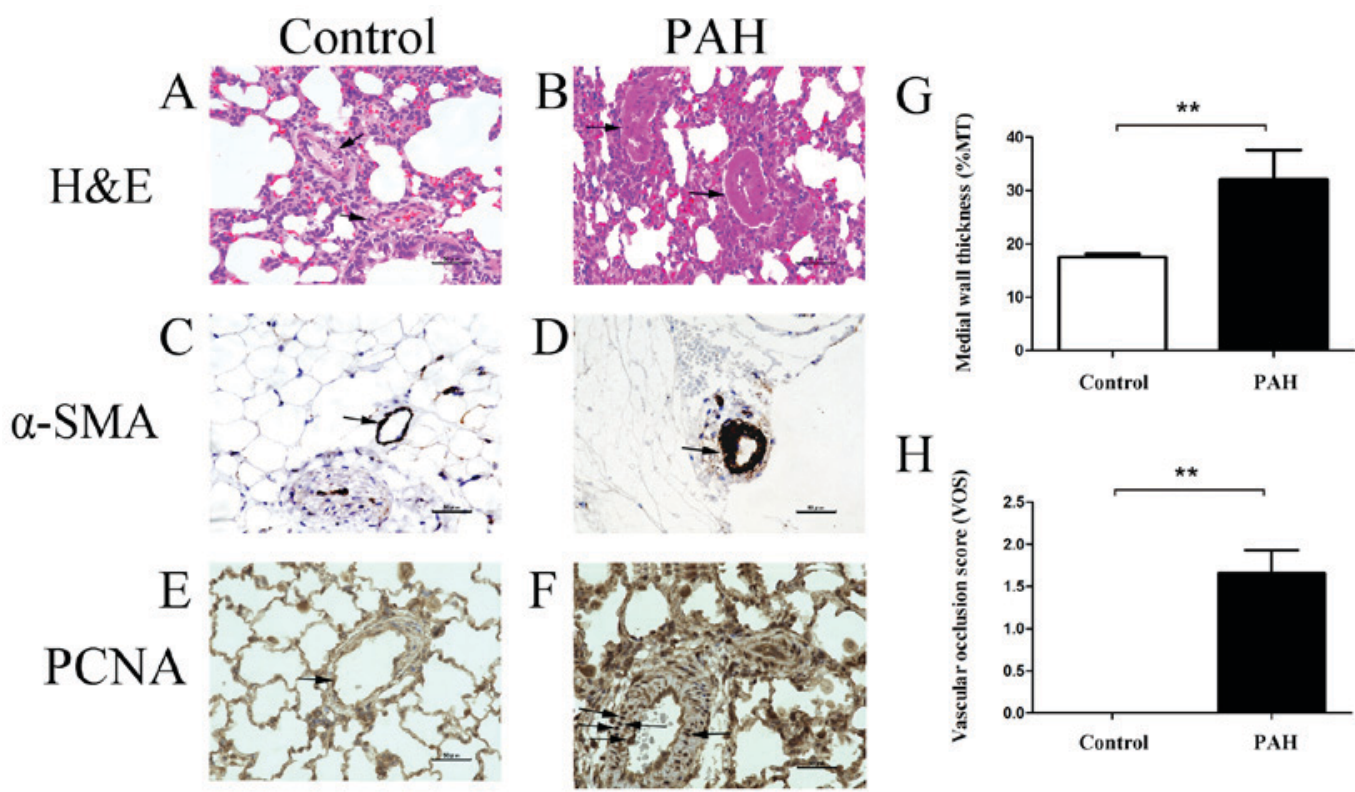

Figure 2. Histological and immunohistochemical evaluation of lung sections in PAH models and normal rats. (A and B) H\&E staining revealed a thicker pulmonary artery wall (arrows) in PAH rats. (C and D) $\alpha$-SMA immunohistochemical staining revealed increased $\alpha$-SMA-positive media wall thickness in PAH rats (arrows). (E and F) PCNA immunohistochemical staining revealed increased proliferation in PAs (arrows) in PAH rats. Scale bar, $50 \mu \mathrm{m}$. (G) Media wall thickness (\%MT) and (H) VOS were calculated and compared between the two groups. ${ }^{* * *} \mathrm{P}<0.01$. PAH, pulmonary arterial hypertension; H\&E, hematoxylin and eosin; $\alpha$-SMA, $\alpha$-smooth muscle actin; PCNA, proliferating cell nuclear antigen; PAs, pulmonary arteries, VOS, vascular occlusion score.

A

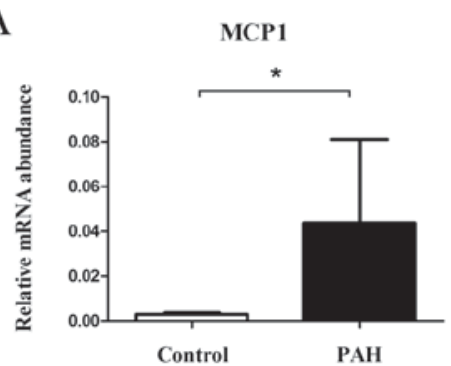

D

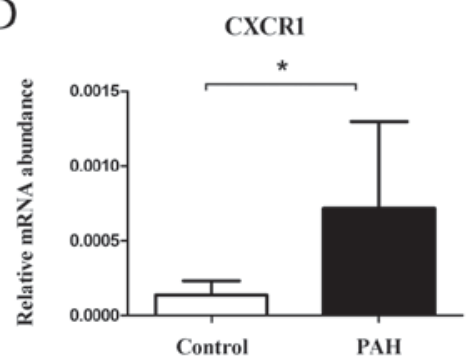

B

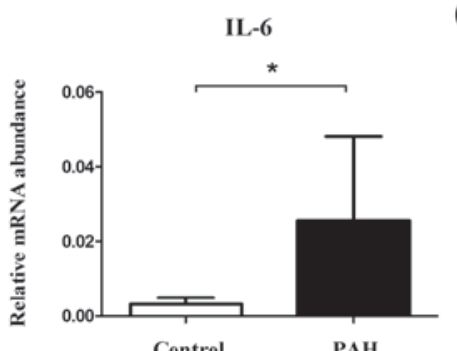

E

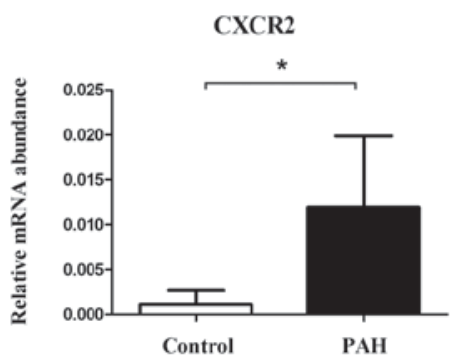

C

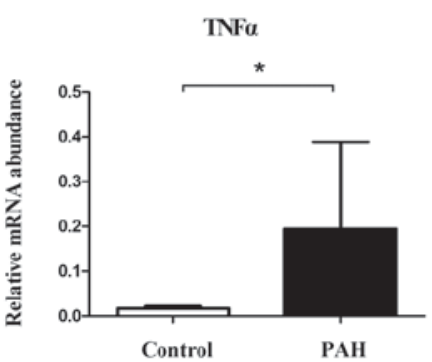

Figure 3. mRNA expression of inflammation genes increased in PAH. (A) MCP1, (B) IL-6, (C) TNF $\alpha$, (D) CXCR1, and (E) CXCR2 mRNA levels increased in PAH animals compared to controls $\left({ }^{*} \mathrm{P}<0.05\right)$. PAH, pulmonary arterial hypertension; MCP1, monocyte chemoattractant protein 1 ; IL-6, interleukin-6; TNFo, tumor necrosis factor $\alpha$; CXCR1, C-X-C chemokine receptor type 1; CXCR2, C-X-C chemokine receptor type 2.

stem cell factor $(\mathrm{SCF})(0.0922 \pm 0.0535$ in the PAH group and $0.0281 \pm 0.0210$ in the control group, $\mathrm{P}=0.012$, Fig. $4 \mathrm{~B}$ ), c-Kit $(0.0205 \pm 0.0071$ in the PAH group and $0.0086 \pm 0.0101$ in the control group, $\mathrm{P}=0.016$, Fig. 4C), and CD29 $(0.0084 \pm 0.0053$ in the PAH group and $0.0027 \pm 0.0015$ in the control group, $\mathrm{P}=0.019$, Fig. 4D).

Protein expression of CXCR4 and other stem cell markers in immunohistochemical staining. To further investigate the expression of CXCR4 and its implications, immunohistochemical staining was performed. Few CXCR4-positive cell clusters were observed in lung tissue of the control group (Fig. 5A). However, there was a marked increase in CXCR4 expression in the PAH group (Fig. 5B), with 10.39 \pm 1.73 CXCR4-positive cell clusters observed in the PAH group compared with $6.83 \pm 1.20$ in the control group ( $\mathrm{P}=0.015$, Fig. 5C). Additionally, compared to the control group (Fig. 5D), c-Kit-positive cells around the PAs were greater in number in the PAH group (Fig. 5E): $31.61 \pm 6.10$ c-Kit-positive cells in the PAH group and $14.43 \pm 3.24$ in 
A

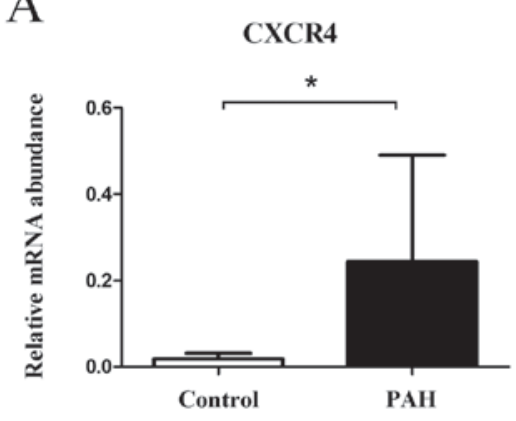

$\mathrm{C}$

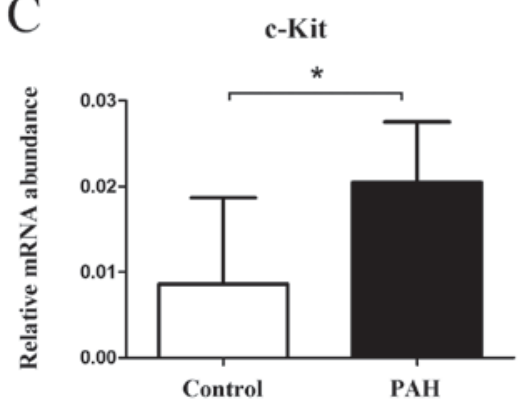

B

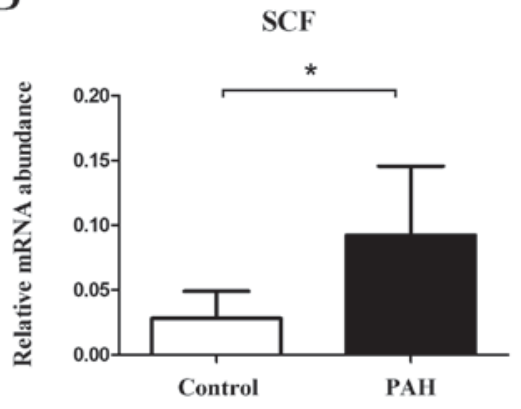

D

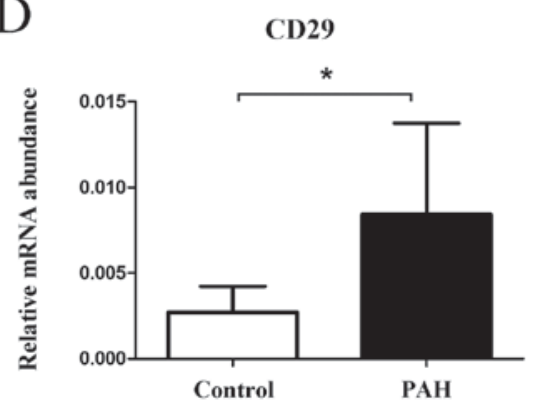

Figure 4. mRNA expression of stem cell genes increased in PAH. (A) CXCR4, (B) SCF, (C) c-Kit, and (D) CD29 mRNA levels increased in PAH animals compared to controls ("P<0.05). PAH, pulmonary arterial hypertension; CXCR4, C-X-C chemokine receptor type 4; SCF, stem cell factor.
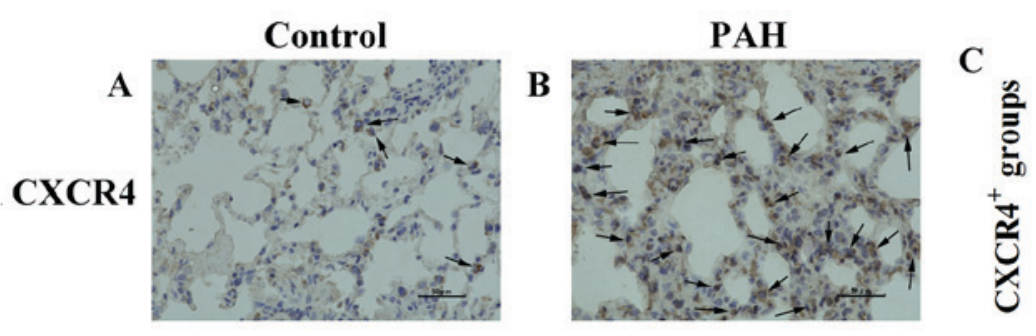

E
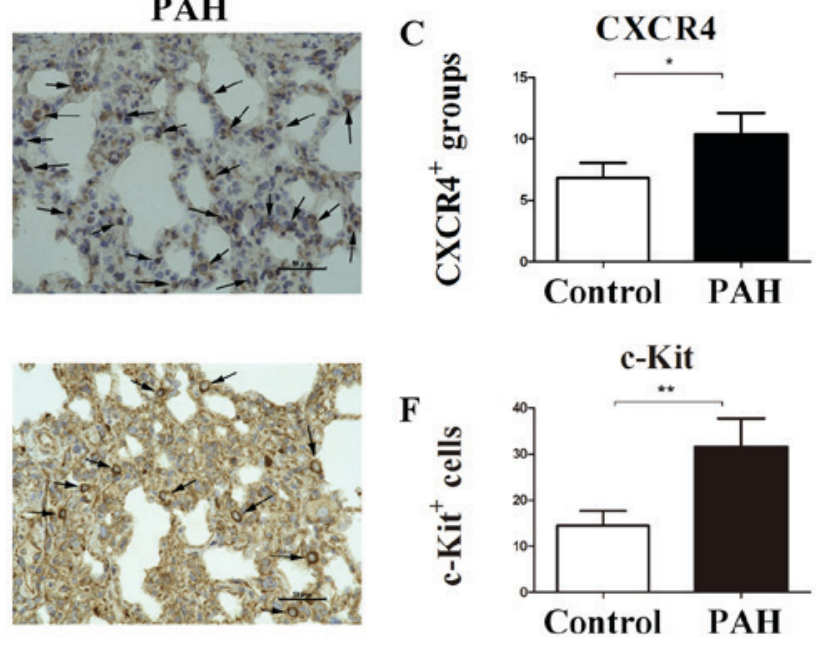

c-Kit
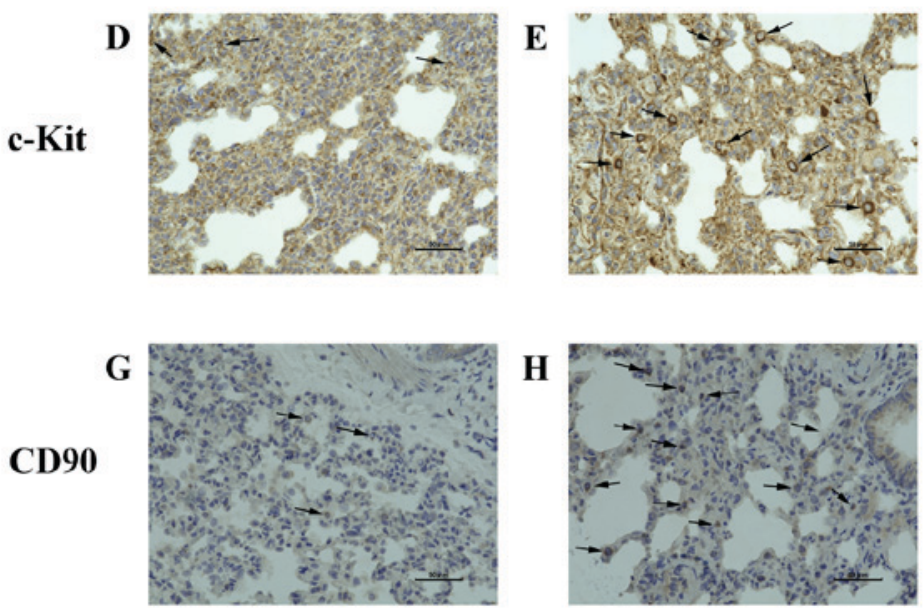

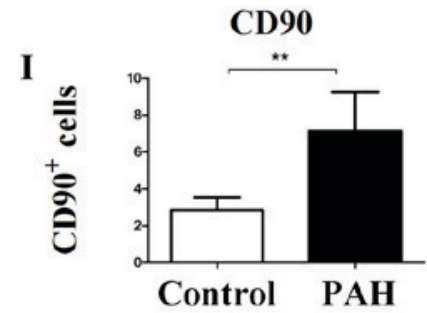

Figure 5. Immunohistochemical evaluation of CXCR4, c-Kit, and CD90 stem cell marker proteins increased in PAH. (A, B and C) Only a few CXCR4-positive cells were observed on vascular cells in the lungs of the control group, but increased CXCR4 expression in PAH group was observed within the lumen and vascular wall of the PAs (arrows). (D, E and F) The number and size of c-Kit-positive cells around PAs were greater in the PAH group (arrows). (G, H and I) The number of CD90-positive cells around PAs was larger in the PAH group (arrows). Scale bar, $50 \mu \mathrm{m} .{ }^{* *} \mathrm{P}<0.01,{ }^{*} \mathrm{P}<0.05$. PAH, pulmonary arterial hypertension; CXCR4, C-X-C chemokine receptor type 4; PAs, pulmonary arteries.

the control group ( $\mathrm{P}=0.003$, Fig. 5F). c-Kit-positive cells were also larger in size in the PAH group. Compared with the control group (Fig. 5G), the number of CD90-positive cells around the PAs was much greater in the PAH group (Fig. $5 \mathrm{H}$ ): $7.13 \pm 2.13$ in the PAH group and $2.85 \pm 0.68$ in the control group ( $\mathrm{P}=0.009$, Fig. 5I). 

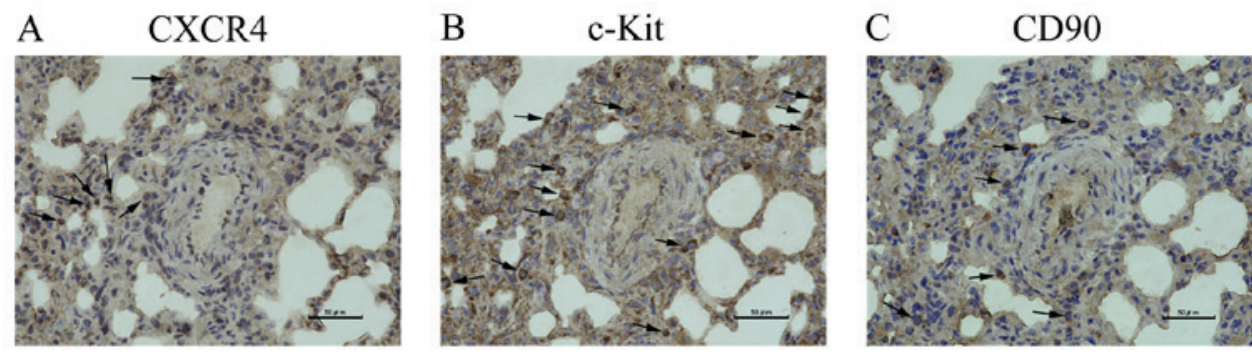

Figure 6. Adjacent serial sections in PAH rats were prepared and used to compare the expression of (A) CXCR4, (B) c-Kit, and (C) CD90. The three proteins differed in their locatioins (arrows). Scale bar, $50 \mu \mathrm{m}$. PAH, pulmonary arterial hypertension; CXCR4, C-X-C chemokine receptor type 4 .

Serial sections were cut from PAH rats to compare the localization of CXCR4 (Fig. 6A), c-Kit (Fig. 6B), and CD90 (Fig. 6C). Differences in the localization of these proteins were observed; protein expression did not overlap.

\section{Discussion}

The main finding of this study is that the expression of CXCR4 and other markers of stem/progenitor cells is significantly higher in PAH rats than in normal rats. Thus, CXCR4 and stem cells such as MSCs may play an important role in pulmonary vascular remodeling. These results provide basic evidence for further studies of treatment with CXCR4 inhibitors for PAH, which may benefit clinical PAH patients.

Methods for establishing PAH models are well-developed. Because the MCT and $\mathrm{CH}$ models are regarded as models of mild pulmonary hypertension (19), a combination of two treatments, such as MCT and $\mathrm{CH}$ (15), MCT and pneumonectomy (20), or Sugen and CH (21), are commonly used to create a severe disease model that mimics human PAH. In the present study, we used a combination of MCT and $\mathrm{CH}$ for experimental simplicity; this method requires only a single subcutaneous injection of MCT and induction of $\mathrm{CH}$, which is straightforward and results in a more severe disease model, which may be more similar to clinical PAH, than a single treatment. We initially developed 6-week models of PAH, but most of the rats died after 5 weeks; therefore, 5-week models were used instead. Significant differences in RVSP and Fulton index between the $\mathrm{PAH}$ and control groups were observed. Additionally, the \%MT values obtained from $\alpha$-SMA immunohistochemical staining showed proliferation of smooth muscle cells in the PAH model, and the VOS (which reflects PCNA-positive proliferating cells) showed pulmonary arteriolar occlusion in the PAH model, as we have described previously (10). Increased expression of MCP1, IL-6, TNF $\alpha$, CXCR1, and CXCR2 mRNA in the PAH model indicated chronic inflammation. These results suggest that we successfully established a PAH model with pulmonary vascular remodeling.

RT-qPCR experiments showed that the expression of CXCR4 and other markers of stem/progenitor cells was significantly higher in PAH rats than in control rats. These results indicate that CXCR4 or stem/progenitor cells play a role in the pathogenesis of PAH. CXCR4 has been shown to be expressed in cancer cells and to contribute to the proliferation and survival of tumor cells (13). We previously showed that valproic acid, an epigenetic modifier, was effective for treating PAH in similar rat models (10). We predicted that CXCR4 plays an important role in the chemotaxis, proliferation, and survival of smooth muscle cells, endothelial cells, and other cells in lung tissue, leading to pulmonary arteriolar occlusion or pulmonary vascular remodeling.

The observation that the expression of stem/progenitor cell markers was higher in the PAH group has several interesting implications. Firstly, c-Kit has been detected on the surface of lung stem cells, which can repopulate airways and vessels (22), and on the surface of lung vascular endothelial stem cells, which generate functional blood vessels (23). SCF, which is the ligand of c-Kit, has also been demonstrated to play an essential role in regulating cell proliferation (24). In our study, c-Kit-positive cells were significantly more numerous and SCF gene expression was significantly enhanced in the PAH group compared with the control group. These c-Kit-positive stem-like cells surrounding blood vessels may differentiate into mature cells and proliferate, eventually contributing to pulmonary vascular remodeling. Secondly, CD90, which is an MSC marker (25), and CD29, which is an MSC and fibroblast marker, were more highly expressed in the PAH group than in the control group, indicating that MSCs are involved in the pathogenesis of PAH; these MSCs can also differentiate into mature cells, proliferate, and contribute to pulmonary vascular remodeling. Thirdly, the serial sections revealed that the localization of CXCR4, c-Kit, and CD90 expression differed, which may be because they have different functions or because a subset of MSCs differentiated along smooth muscle or endothelial lineages as part of the PAH injury-repair response.

Interestingly, Farkas et al found that the number of c- $\mathrm{Kit}^{+}$ $\mathrm{vWF}^{+}$cells and the expression of CXCL12 began decreasing after 21 days in a Sugen and CH-induced rat model of PAH (14). Based on these data and the present study, we propose that in the first three weeks of progression in rat models of PAH, stem cells emerge and begin to proliferate, constituting a protective and compensatory mechanism against $\mathrm{PAH}$, such that the rat does not initially show symptoms of heart failure. However, as pulmonary arterial pressure continues to increase, the compensatory mechanism becomes insufficient, symptoms begin to occur, and stem cells stop growing and begin to decline in number; however, pulmonary vascular remodeling may have already occurred because of the stem cells and through other mechanisms. Hence, if PAH rats or patients could be treated with a CXCR4 inhibitor while the compensatory mechanism is still effective, fewer CXCR4-positive cells would emerge and pulmonary vascular remodeling may be less severe.

Our experimental results and previous studies $(26,27)$ suggest that CXCR4 is involved in PAH development; thus, 
CXCR4 inhibitors may be a potential treatment for PAH. Although one small-molecule CXCR4 inhibitor has already been reported to prevent pulmonary arterial muscularization in a PAH model $(14,28)$, the role of CXCR4 in the pathogenesis and progression of PAH and the potential of other CXCR4 inhibitors such as LY2510924 or T134 to prevent PAH remain unclear. CXCR4 inhibitors currently in clinical trials are used predominantly for various cancers $(29,30)$ and HIV therapy (31). The use of CXCR4 inhibitors in PAH treatment may decrease the number of CXCR4-positive stem cells, decreasing the number of mature cells such as smooth muscle cells and endothelial cells, thereby reducing proliferation, vascular occlusion, and vascular remodeling. CXCR4 inhibitors may therefore offer an alternative to the three major drug classes currently in clinical use for PAH treatment and to organ transplantation, providing new options for $\mathrm{PAH}$ patients.

The present study has some limitations. Firstly, animal models are less complex than clinical patients, and it is difficult to establish congenital or hereditary PAH models. Secondly, although we confirmed the expression of CXCR4 and other proteins, we did not confirm the efficacy of inhibitors of these proteins in PAH treatment. Thirdly, CXCR4 has a complex biological function; CXCR4-expressing cells can be recruited to SDF-1-expressing cells in injured areas, assisting injury repair (32). Therefore, further studies of CXCR4 are necessary. Finally, the precise role of stem/progenitor cells in PAH should be examined in more detail.

\section{Acknowledgements}

The authors acknowledge Mr. Kenji Yoshihara and Mr. Hiroaki Nagao at Tokyo Women's Medical University for their technical assistance.

\section{Funding}

The authors would like to acknowledge the China Scholarship Council (grand no. 201708050127) for financially supporting the studies of $\mathrm{TZ}$ as a foreign student.

\section{Availability of data and materials}

The datasets used and/or analyzed during the current study are available from the corresponding author on reasonable request.

\section{Authors' contributions}

TZ and TN designed the concept and conducted the experiments. TZ constructed the animal models, performed RVSP measurements and wrote the paper. NK performed data analysis and modified the paper. EH performed reverse transcription-quantitative polymerase chain reaction. YF performed the immunohistochemical staining. TN was also involved in mechanism analysis.

\section{Ethics approval and consent to participate}

All experiments were conducted according to a protocol approved by the Institutional Animal Experiment Committee of the Tokyo Women's Medical University (AE16-117).

\section{Consent for publication}

Not applicable.

\section{Competing interests}

The authors declare that they have no competing interests.

\section{References}

1. Zhou L, Chen Z, Vanderslice P, So SP, Ruan KH, Willerson JT and Dixon RA: Endothelial-like progenitor cells engineered to produce prostacyclin rescue monocrotaline-induced pulmonary arterial hypertension and provide right ventricle benefits. Circulation 128: 982-994, 2013.

2. Cheng GS, Zhang YS, Zhang TT, He L and Wang XY: Bone marrow-derived mesenchymal stem cells modified with IGFBP-3 inhibit the proliferation of pulmonary artery smooth muscle cells. Int J Mol Med 39: 223-230, 2017.

3. Farber HW and Loscalzo J: Pulmonary arterial hypertension. $\mathrm{N}$ Engl J Med 351: 1655-1665, 2004.

4. Huertas A, Perros F, Tu L, Cohen-Kaminsky S, Montani D, Dorfmüller P, Guignabert C and Humbert M: Immune dysregulation and endothelial dysfunction in pulmonary arterial hypertension: A complex interplay. Circulation 129: 1332-1340, 2014.

5. Tuder RM, Archer SL, Dorfmüller P, Erzurum SC, Guignabert C, Michelakis E, Rabinovitch M, Schermuly R, Stenmark KR and Morrell NW: Relevant issues in the pathology and pathobiology of pulmonary hypertension. J Am Coll Cardiol 62 (25 Suppl): D4-D12, 2013.

6. Ranchoux B, Antigny F, Rucker-Martin C, Hautefort A, Péchoux C, Bogaard HJ, Dorfmüller P, Remy S, Lecerf F, Planté S, et al: Endothelial-to-mesenchymal transition in pulmonary hypertension. Circulation 131: 1006-1018, 2015.

7. Yang JX, Pan YY, Zhao YY and Wang XX: Endothelial progenitor cell-based therapy for pulmonary arterial hypertension. Cell Transplant 22: 1325-1336, 2013.

8. Chen H, Strappe P, Chen S and Wang LX: Endothelial progenitor cells and pulmonary arterial hypertension. Heart Lung Circ 23: 595-601, 2014.

9. Wang XX, Zhang FR, Shang YP, Zhu JH, Xie XD, Tao QM, Zhu JH and Chen JZ: Transplantation of autologous endothelial progenitor cells may be beneficial in patients with idiopathic pulmonary arterial hypertension: A pilot randomized controlled trial. J Am Coll Cardiol 49: 1566-1571, 2007.

10. Lan B, Hayama E, Kawaguchi N, Furutani Y and Nakanishi T: Therapeutic efficacy of valproic acid in a combined monocrotaline and chronic hypoxia rat model of severe pulmonary hypertension. PLoS One 10: e0117211, 2015.

11. Teicher BA and Fricker SP: CXCL12 (SDF-1)/CXCR4 pathway in cancer. Clin Cancer Res 16: 2927-2931, 2010.

12. Miller RJ, Banisadr G and Bhattacharyya BJ: CXCR4 signaling in the regulation of stem cell migration and development. J Neuroimmunol 198: 31-38, 2008.

13. Peng SB, Zhang X, Paul D, Kays LM, Gough W, Stewart J, Uhlik MT, Chen Q, Hui YH, Zamek-Gliszczynski MJ, et al: Identification of LY2510924, a novel cyclic peptide CXCR4 antagonist that exhibits antitumor activities in solid tumor and breast cancer metastatic models. Mol Cancer Ther 14: 480-490, 2015.

14. Farkas D, Kraskauskas D, Drake JI, Alhussaini AA, Kraskauskiene V, Bogaard HJ, Cool CD, Voelkel NF and Farkas L: CXCR4 inhibition ameliorates severe obliterative pulmonary hypertension and accumulation of C-kit ${ }^{+}$cells in rats. PLoS One 9: e89810, 2014.

15. Morimatsu Y, Sakashita N, Komohara Y, Ohnishi K, Masuda H, Dahan D, Takeya M, Guibert C and Marthan R: Development and characterization of an animal model of severe pulmonary arterial hypertension. J Vasc Res 49: 33-42, 2012.

16. Rondelet B, Kerbaul F, Motte S, van Beneden R, Remmelink M, Brimioulle S, McEntee K, Wauthy P, Salmon I, Ketelslegers JM and Naeije R: Bosentan for the prevention of overcirculation-induced experimental pulmonary arterial hypertension. Circulation 107: 1329-1335, 2003.

17. Nishimura T, Vaszar LT, Faul JL, Zhao G, Berry GJ, Shi L, Qiu D, Benson G, Pearl RG and Kao PN: Simvastatin rescues rats from fatal pulmonary hypertension by inducing apoptosis of neointimal smooth muscle cells. Circulation 108: 1640-1645, 2003. 
18. Bahmanpour S, Talaei Khozani T, Zarei Fard N, Jaberipour M, Hosseini A and Esmaeilpour T: A comparison of the multiple oocyte maturation gene expression patterns between the newborn and adult mouse ovary. Iran J Reprod Med 11: 815-822, 2013.

19. Stenmark KR, Meyrick B, Galie N, Mooi WJ and McMurtry IF: Animal models of pulmonary arterial hypertension: The hope for etiological discovery and pharmacological cure. Am J Physiol Lung Cell Mol Physiol 297: L1013-L1032, 2009.

20. Okada K, Tanaka Y, Bernstein M, Zhang W, Patterson GA and Botney MD: Pulmonary hemodynamics modify the rat pulmonary artery response to injury. A neointimal model of pulmonary hypertension. Am J Pathol 151: 1019-1025, 1997.

21. Abe K, Toba M, Alzoubi A, Ito M, Fagan KA, Cool CD, Voelkel NF, McMurtry IF and Oka M: Formation of plexiform lesions in experimental severe pulmonary arterial hypertension. Circulation 121: 2747-2754, 2010.

22. Kajstura J, Rota M, Hall SR, Hosoda T, D'Amario D, Sanada F, Zheng H, Ogórek B, Rondon-Clavo C, Ferreira-Martins J, et al: Evidence for human lung stem cells. N Engl J Med 364: 1795-1806, 2011.

23. Fang S, Wei J, Pentinmikko N, Leinonen $H$ and Salven $P$ : Generation of functional blood vessels from a single c-kit+ adult vascular endothelial stem cell. PLoS Biol 10: e1001407, 2012.

24. Kim JY, Choi JS, Song SH, Im JE, Kim JM, Kim K, Kwon S, Shin HK, Joo CK, Lee BH and Suh W: Stem cell factor is a potent endothelial permeability factor. Arterioscler Thromb Vasc Biol 34: 1459-1467, 2014.

25. Ridzuan N, Al Abbar A, Yip WK, Maqbool M and Ramasamy R: Characterization and expression of senescence marker in prolonged passages of rat bone marrow-derived mesenchymal stem cells. Stem Cells Int 2016: 8487264, 2016.

26. Huang X, Wu P, Huang F, Xu M, Chen M, Huang K, Li GP, Xu M, Yao D and Wang L: Baicalin attenuates chronic hypoxia-induced pulmonary hypertension via adenosine A2A receptor-induced SDF-1/CXCR4/PI3K/AKT signaling. J Biomed Sci 24: 52, 2017.
27. Favre S, Gambini E, Nigro P, Scopece A, Bianciardi P, Caretti A, Pompilio G, Corno AF, Vassalli G, von Segesser LK, et al: Sildenafil attenuates hypoxic pulmonary remodelling by inhibiting bone marrow progenitor cells. J Cell Mol Med 21: 871-880, 2017.

28. Young KC, Torres E, Hatzistergos KE, Hehre D, Suguihara C and Hare JM: Inhibition of the SDF-1/CXCR4 axis attenuates neonatal hypoxia-induced pulmonary hypertension. Circ Res 104: 1293-1301, 2009.

29. Ling X, Spaeth E, Chen Y, Shi Y, Zhang W, Schober W, Hail N Jr, Konopleva M and Andreeff M: The CXCR4 antagonist AMD3465 regulates oncogenic signaling and invasiveness in vitro and prevents breast cancer growth and metastasis in vivo. PLoS One 8: e58426, 2013.

30. Galsky MD, Vogelzang NJ, Conkling P, Raddad E, Polzer J, Roberson S, Stille JR, Saleh M and Thornton D: A phase I trial of LY2510924, a CXCR4 peptide antagonist, in patients with advanced cancer. Clin Cancer Res 20: 3581-3588, 2014.

31. Arakaki R, Tamamura H, Premanathan M, Kanbara K, Ramanan S, Mochizuki K, Baba M, Fujii N and Nakashima H: T134, a small-molecule CXCR4 inhibitor, has no cross-drug resistance with AMD3100, a CXCR4 antagonist with a different structure. J Virol 73: 1719-1723, 1999.

32. Yang JX, Zhang N, Wang HW, Gao P, Yang QP and Wen QP: CXCR4 receptor overexpression in mesenchymal stem cells facilitates treatment of acute lung injury in rats. J Biol Chem 290: 1994-2006, 2015.

This work is licensed under a Creative Commons Attribution-NonCommercial-NoDerivatives 4.0 International (CC BY-NC-ND 4.0) License. 